\section{MS15-P35 Relationship between the composition, structural parameters and properties of single-crystal KDP with nano-titania}

Galina M. Kuzmicheva ${ }^{1}$, Olesya I. Timaeva ${ }^{1}$, Elena N. Domoroshchina $^{1}$, Vadim V. Grebenev ${ }^{2}$, Anna V. Kosinova ${ }^{3}$

1. Department of Materials Science and Technology of Functional Materials and Structures, State University of Fine Chemical Technologies of M. V. Lomonosov, 119571, Moscow, Russia

2. Institute of Crystallography, Russian Academy of Sciences, 119333, Moscow, Russia

3. Institute for Single Crystals NAS of Ukraine, 61001, 60 Lenin Ave. Kharkov, Ukraine

email: galina_kuzmicheva@list.ru

Development perspectives for new nonlinear optical elements (NLO) based on combinations of inorganic matrix with various inorganic functional nanoparticles are discussed now. The presence of nano- $\mathrm{TiO}_{2}$ in a crystalline matrix of KDP ( $\left.\mathrm{KH}_{2} \mathrm{PO}_{4}\right)$ leads to increase the magnitude of cubic nonlinear susceptibility, changes the sign of the nonlinear refractive response and increases the efficiency of second harmonic generation of composite system KDP:TiO (V.Ya. Gayvoronsky et al. 2013). The aim of this paper is to establish the relationship between composition, structural parameters and dielectric properties of composites $\mathrm{KDP}: \mathrm{TiO}_{2}$.

X-ray diffraction study of the initial samples with nano- $\mathrm{TiO}_{2}$ (phase analysis, the sizes of coherent scattering regions - $D$ ) indicated the presence of anatase (sample $1 ; D=50(4) \AA$ ) and $\eta-\mathrm{TiO}$ (sample $3 ; D=38$ (2) A) (sulfate method) and anatase (sample 2; $D=150$ (8) A) (chloride method) at them. Pure KDP crystals and KDP:TiO (composites I, II, III with incorporated $\mathrm{TiO}_{2}$ nanoparticles from the samples 1,2 and 3, correspondingly) were grown by the temperature reduction method onto point seed. According to X-ray microanalysis, the sulfur content is greater in the sample 3 (4.37-7.58 wt \%) and composite III (11.99 wt.\%) as compared with sample 1 (2.17-3.73 wt \%) and composite I (4.54 wt..\%). The samples from growth sectors $\{100\}$ and $\{101\}$ of KDP and $\mathrm{KDP}: \mathrm{TiO}_{2}$ crystals were cut for the investigation.

Analysis of the results of X-ray single crystal study revealed the most significant structural changes in the composite III $(\{100\})$ : a very small value of $\mathrm{O}-\mathrm{H}$ distance and a short distance P-O compared with the same distances in the structures of KDP and composites I, II, vacancies in the $\mathrm{K}^{1+}$ sites and located ions $\mathrm{Ti}^{4+}$ in the vicinity of the $\mathrm{K}^{1+}$ positions. It was established that the general composition of the composite III $(\{100\})$ can be ]$\left._{\mathrm{x}}\right)\left[\left(\mathrm{PO}_{4}\right)^{3-}\left(\mathrm{SO}_{4}{ }^{2-}\right)_{1-\mathrm{y}}\right]\left(\mathrm{K}_{0.950(1)}[]_{0.050}\right)\left(\mathrm{Ti}_{0.052(2) \mathrm{i}}\right)\left(\mathrm{H}^{1+}{ }_{2-\mathrm{x}}[\right.$ It was established that the magnitude of dielectric permittivity $\left(\varepsilon^{\prime}\right)$ for KDP and $\mathrm{KDP}: \mathrm{TiO}_{2}$ is different depending on the growth sectors $\left(\varepsilon^{\prime}\{101\}>\varepsilon^{\prime}\{100\}\right)$. It is greater for composite II in comparison with composite I and composite III $(\{100\})$ has the smallest value of $\varepsilon^{\prime}$, which correlates with the interatomic distance $\mathrm{O}-\mathrm{H}$.

This work was carried out as a part of a state task of the Ministry of Education and Science of Russian Federation (№ 4.745.2014/K; 2014-2016).

\author{
MS15-P36 Absolute structure of (E \\ )-2,2'-[3-(2-Nitrophenyl)prop-2-ene-1,1-diyl] \\ bis (3-hydroxy- \\ 5,5-dimethylcyclohex-2-en-1-one) \\ Joo Hwan Cha ${ }^{1}$, Jae Kyun $\mathrm{Lee}^{2}$, Yong Seo $\mathrm{Cho}^{2}$
}

1. Advanced Analysis Center, Korea Institute of Science \& Technology, Hwarangro 14-gil, Seongbuk-gu, Seoul, South Korea 2. Center for Neuro-Medicine, Korea Institute of Science \& Technology, Hwarangro 14-gil, Seongbuk-gu, Seoul, South Korea

email: jhcha@kist.re.kr

Herewith we present the crystal structure of (E)-2,2'-[3-(2-nitrophenyl)prop-2-ene-1,1-diyl]

bis(3-hydroxy-5,5-dimethylcyclohex-2-en-1-one) (A)[1], (E)-2,2'-[3-(4-nitrophenyl)prop-2-ene-1,1-diyl]

bis(3-hydroxy-5,5-dimethylcyclohex-2-en-1-one) (B)[2]. In the compound (A), $\mathbf{C} 25 \mathrm{H} 29 \mathrm{NO} 6$, each of the cyclohexenone rings adopts a half-chair conformation. Each of the pairs of hydroxy and carbonyl $\mathrm{O}$ atoms are oriented to allow for the formation of intramolecular $\mathrm{O}-\mathrm{H}---\mathrm{O}$ hydrogen bonds, which are typical of xanthene derivatives. The nitro group is rotationally disordered over two orientations in a $0.544(6): 0.456$ (6) ratio. In the crystal, weak intermolecular $\mathrm{C}-\mathrm{H}---\mathrm{O}$ hydrogen bonds link molecules into layers parallel to the $a b$ plane. The compound (B), each of the cyclohexenone rings adopts a half-chair conformation. The hydroxy and carbonyl $\mathrm{O}$ atoms face each other and are oriented to allow for the formation of two intramolecular $\mathrm{O}-\mathrm{H}---\mathrm{O}$ hydrogen bonds. In the crystal, weak $\mathrm{C}-\mathrm{H}---\mathrm{O}$ hydrogen bonds are formed between molecules, generating a two-dimensional supramolecular structure.

[1] Cha, J, H., Kim, Y. H., Min, S. J., Cho, Y. S. \& Lee, J. K. (2011). Acta Cryst. E67, o3153.

[2] Cha, J, H., Cho, Y. S.,Lee, J. K., Park, J. H. \& Sato, H. (2012). Acta Cryst. E68, o2510.

Keywords: X-ray crystallography of organic compounds; xanthene; absolute structure determination 